\title{
ROBUST INTERFERENCE SUPPRESSION FOR MULTICHANNEL SAR
}

\author{
Luke Rosenberg ${ }^{a, b, c}$ and Doug Gray ${ }^{a, b}$
}

(a) Department of Electrical and Electronic Engineering, University of Adelaide, Australia

(b) Cooperative Research Centre for Sensor, Signal and Information Processing (CSSIP)

(c) Defence, Science and Technology Organisation (DSTO)

luker@eleceng.adelaide.edu.au, dgray@eleceng.adelaide.edu.au

\begin{abstract}
Forming a Synthetic Aperture Radar (SAR) image while suppressing a broadband jammer can potentially destroy large regions of the image. In addition to this, multipath reflections from the ground, known as hot-clutter or terrain scattered interference will add a non-stationary interference component to the image. The goal of interference suppression for SAR is to successfully suppress these interferences while not significantly effecting the image quality by blurring, reducing the resolution or raising the side-lobe level. Using multiple antennas on a SAR provides spatial degrees of freedom and allows for adaptive beamforming to suppress the jammer signals. This paper presents two constrained spatial techniques which reduce the interference level without significantly effecting the image quality.
\end{abstract}

\section{INTRODUCTION}

Typical SAR imaging is performed with a large offset range and small field of view. Any jammer signal incident outside the main-beam field of view can easily be suppressed with spatial beamforming alone. However, if the jammer signal is incident in the main-beam, the range profile of a target can be nulled and consequent image formation will lead to a blurry final image. In addition to this, nonstationary interference from the 'hot-clutter' will cause the training statistics to change from pulse to pulse and traditional slow-time Space Time Adaptive Processing (STAP) techniques [1], will not be effective. Therefore adapting within each pulse is required by exploiting spatial beamforming or combining spatial/fast-time beamforming.

For the similar problem of detecting moving targets in airborne radar, a number of space/fast-time approaches have been suggested, [2]. The secondary problem known as 'coherency modulation' has also been been studied in [3]. To begin addressing this problem for SAR, this paper is restricted to spatial only adaption and presents two 'robust' methods which restore the final image quality by using either derivative or amplitude constraints. As experimental data was not available, results are based on simulations using a diffuse scattering model.

\section{SYSTEM MODELS AND GEOMETRY}

Consider a SAR travelling along the $\mathrm{y}$-axis, imaging a patch in the slant-plane $x \in\left[X_{c}-X_{0}, X_{c}+X_{0}\right], y \in$ $\left[-Y_{0}, Y_{0}\right]$. The radar transmits a broadband chirp and the received signal is base-banded and sampled for each of the $N$ channels of a linear antenna array with equi-spaced receivers in the azimuth direction. The signal model for the $n^{\text {th }}$ channel is given by,

$$
\begin{gathered}
\tilde{s}_{n}(t, u, x, y)=a(t) \exp \left[-j \omega_{c} \tau_{n}(x, y-u)+\right. \\
\left.j \alpha\left(t-\tau_{n}(x, y-u)\right)^{2}\right]
\end{gathered}
$$

where the chirp pulse duration $T_{p}$ is defined by $a(t)$ which is unity for $0 \leq t \leq T_{p}$ and zero otherwise. The carrier frequency is given by $\omega_{c}(\mathrm{rad} / \mathrm{s})$ and the chirp rate by $\alpha$ $(\mathrm{rad} / \mathrm{s})$. The variables $(t, u)$ represent (fast-time) samples within a pulse and the SAR platform position (slow-time) respectively. If a signal is transmitted from the centre of the linear array when the SAR platform is at $u$, then the relative delay for the $n^{\text {th }}$ channel is given by the sum of temporal and spatial delays,

$$
\tau_{n}(x, y-u)=\frac{1}{c}\left[R\left(x, y-u-d_{n}\right)+R(x, y-u)\right]
$$

where $R(\cdot)$ is the radial distance, $d_{n}=n \delta$ is the antenna offset from the array phase centre with antenna spacing $\delta$ and $n \in[-(N-1) / 2,(N-1) / 2]$ for $N$ (odd) antenna elements. As the SAR bandwidth, $B(\mathrm{~Hz})$ is much smaller than the carrier frequency, $\omega_{c}(\mathrm{rad} / \mathrm{s})$, the SAR signal model can be split into temporal and spatial components. The spatial component of the total delay is given by the time difference between the centre and the $n^{\text {th }}$ channel. It can be approximated as a function of just the SAR position $u$ or equivalently, an angular offset $\theta(u)$.

$$
\begin{aligned}
\tilde{\tau}_{n}(u) & =\frac{1}{c}\left[R\left(X_{c}, u+d_{n}\right)-R\left(X_{c}, u\right)\right] \\
& \approx \frac{d_{n}}{c} \sin \underbrace{\left(\arctan \left(u / X_{c}\right)\right)}_{\theta(u)} .
\end{aligned}
$$

The spatial steering vector can then be written as

$$
s_{n}(u)=\exp \left[-j \omega_{c} \tilde{\tau}_{n}(u)\right]
$$

and the corresponding SAR signal model as

$$
\begin{array}{r}
\tilde{s}_{n}(t, u, x, y) \approx a(t) \exp \left[-j \omega_{c} \tau(x, y-u)+\right. \\
\left.j \alpha(t-\tau(x, y-u))^{2}\right] s_{n}(u)
\end{array}
$$


The separation of temporal and spatial components is a key point and implies that spatial adaptive filtering can occur independent of fast-time and either before or after range processing if desired.

The total ground return for the SAR is the integral over all scatterers with radar cross section $f(x, y)$,

$$
\gamma_{n}(t, u)=\int_{y} \int_{x} f(x, y) \tilde{s}_{n}(t, u, x, y) d x d y .
$$

Also, if the SAR is being jammed by an airborne platform, there will be an extra signal component required in the data model to represent the direct-path and the ground reflected path (hot-clutter), $z_{n}(\cdot)$. These signals with the addition of receiver noise $\nu(\cdot)$, form the components seen by the SAR. ${ }^{1}$

$$
x_{n}(t, u)=\gamma_{n}(t, u)+z_{n}(t, u)+\nu_{n}(t, u)
$$

The noise signal $\nu_{n}(\cdot)$ represents the receiver noise for each channel. It is modelled as white Gaussian noise with zero mean and unity variance. Figure 1 shows the processing chain from transmission of the chirp signal, formation of the signal $x_{n}(t, u)$, adaption, range processing and image formation.

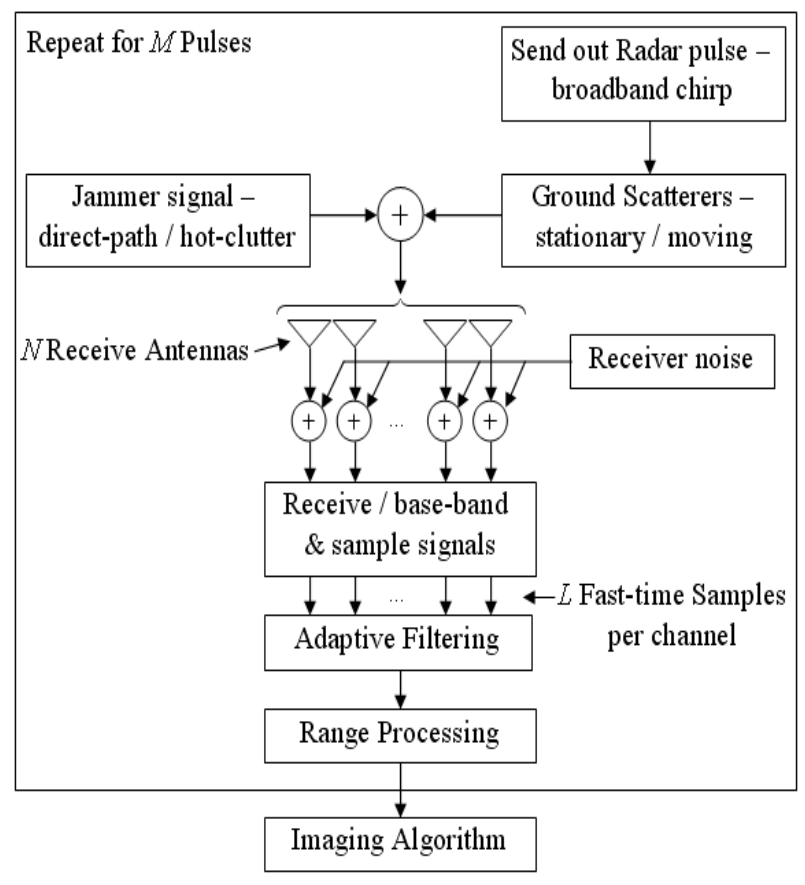

Fig. 1. SAR Processing Diagram

\subsection{Jammer model}

The bistatic jammer model assumes there are $K$ hot-clutter patches within a given area. The position and velocity of the $k^{t h}$ patch relative to the SAR and jammer platforms is shown in Figure 2.

\footnotetext{
${ }^{1}$ Note: If there were moving targets in the scene, these would be additional components.
}

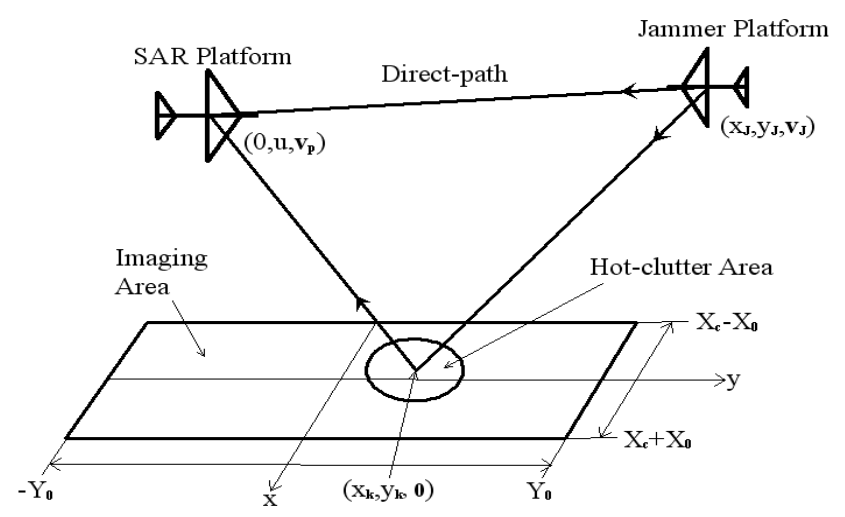

Fig. 2. Jammer Geometry

If an absolute time variable, $\tilde{t}=u / v_{p}+t$ is defined as the sum of slow-time and fast-time, then the output of the $n^{t h}$ receiver, $z_{n}(\cdot)$, is the superposition of the direct path and the delayed reflectors for each patch,

$$
z_{n}(t, u)=\sum_{k=0}^{K} b_{k} J\left(\tilde{t}-\tilde{\tau}_{n, k}(t, u)\right)
$$

where $J(\cdot)$ is the jamming signal waveform, $\tilde{\tau}_{n, k}(\cdot)$ is the bistatic delay and $b_{k}$ is defined as the relative magnitude between the direct-path signal and the jammer signal reflected by the $k^{t h}$ scatterer. The zero index refers to the direct-path with $b_{0}=1$.

The power spectral density of the jammer signal has a bandwidth $B \ll \omega_{c}$, centred at baseband. Although the jammer bandwidth is greater than the SAR bandwidth, the received signal is filtered within the receiver front-end to match the SAR bandwidth. Realisations of the jammer signal $J(\cdot)$ can be created from the Inverse Fourier Transform of this signal or autocorrelation,

$$
r_{J}(\tau)=\operatorname{sinc}(\pi B \tau)
$$

A physically based model for the multipath scattering is presented by Beckman, [4] and used by [5]. It uses a flatearth approximation and is referred to as a glistening surface. Using this model, a surface roughness parameter $K_{\beta}$ defines the scattering distribution between the SAR and an airborne jammer at heights $h_{P}$ and $h_{J}$ respectively, separated by a distance $\tilde{x}_{J}$ in the ground plane. The scatterer positions are projected onto the slant plane to fit our simulation model and rotated by $\theta_{J}$ according to the jammer position. The coefficients, $b_{k}=\rho B_{k}$ for $k>1$ are formed with a scaling factor $\rho$, relative to the direct-path and a random magnitude $B_{k}$, determined from the scattering model.

The degree of diffuseness from the hot-clutter will greatly affect the final image quality. For example, a high $K_{\beta}$ will cause the hot-clutter reflections to be specular and it will appear spatially that only one jamming source is present. However, when $K_{\beta}$ is low, the diffuseness is large and the hot-clutter will spread in angle, giving the appear- 
ance of a number of different jammer sources incident on the SAR.

For the analysis in this paper, a multichannel SAR simulation has been implemented in MATLAB. The parameters chosen are summarised in Table 1 and a comparison between a synthetic SAR ' $S$ ' image and the same image with hot-clutter added is shown in Figure 3. The jammer has been applied in the mainbeam and the diffuse hot-clutter scatterers are both inside and outside the mainbeam. For image formation, a multichannel Spatial Matched Filter / Interpolation algorithm is used [6].

Table 1. Simulation Parameters

\begin{tabular}{|l|l|}
\hline Parameters & Value \\
\hline Carrier Frequency $\left(f_{c}\right) /$ Bandwidth $(B)$ & $10 / 0.3 \mathrm{GHz}$ \\
Number of Elements $(N) /$ Spacing $(\delta)$ & $5 / \frac{\lambda}{2} \mathrm{~m}$ \\
Number of Pulses $(M) /$ Range Bins $(L)$ & $200 / 150$ \\
Range $\left(\Delta_{X}\right) /$ Azimuth Resolution $\left(\Delta_{Y}\right)$ & $1 / 5 \mathrm{~m}$ \\
Range Center $\left(X_{c}\right) /$ Targ. Power $\left(\sigma_{p}^{2}\right)$ & $10 \mathrm{~km} / 20 \mathrm{~dB}$ \\
SAR Height $\left(h_{P}\right) /$ Jammer Height $\left(h_{J}\right)$ & $3 / 3 \mathrm{~km}$ \\
Surface Roughness $\left(K_{\beta}\right) /$ Jam. Offset $\left(\tilde{x}_{J}\right)$ & $0.1 / 50 \mathrm{~km}$ \\
Velocity - SAR $\left(v_{P}\right) /$ Jammer $\left(v_{J}\right)$ & $200 / 100 \mathrm{~m} / \mathrm{s}$ \\
Direct Path Jam. - Power $\left(\sigma_{J}^{2}\right) /$ Angle $\left(\theta_{J}\right)$ & $70 \mathrm{~dB} / 0 \mathrm{deg}$ \\
No. HC Scats. $(K) /$ Relative HC power $(\rho)$ & $200 /-10 \mathrm{~dB}$ \\
\hline
\end{tabular}

\section{CONSTRAINED FILTERING}

To focus an array of receivers, a spatial steering vector is required to determine the response for each focussing position. If we form vectors from the $N$ channels of the received signal $x_{n}(t, u)$ and the spatial steering vector $s_{n}(u)$ from Equation 2,

$$
\begin{aligned}
\mathbf{x}(t, u) & =\left[x_{1}(t, u), \ldots, x_{N}(t, u)\right]^{T} \in \mathcal{C}^{N \times 1} \\
\mathbf{s}(u) & =\left[s_{1}(u), \ldots, s_{N}(u)\right]^{T} \in \mathcal{C}^{N \times 1}
\end{aligned}
$$

then the focussed or beamformed conventional output is given by,

$$
y(t, u)=\mathbf{s}^{H}(u) \mathbf{x}(t, u)
$$

To use an adaptive algorithm, the steering vector is replaced with a weight vector $\mathbf{w}(u)$ designed to minimise the mean square value of the weighted observation subject to the constraint $\mathbf{C}^{H}(u) \mathbf{w}(u)=\mathbf{d}$. $\mathbf{C}(u)$ is the constraint matrix and $\mathbf{d}$ is a column matrix of constraining values. The output power of the general optimisation problem can be formally stated as

$$
\underset{\mathbf{w}(u)}{\min } E\left\{\left|\mathbf{w}^{H}(u) \mathbf{x}(t, u)\right|^{2}\right\} \text { subject to } \mathbf{C}^{H}(u) \mathbf{w}(u)=\mathbf{d}
$$

where the output power can be written in terms of the covariance matrix, $E\left\{\left|\mathbf{w}^{H}(u) \mathbf{x}(t, u)\right|^{2}\right\}=\mathbf{w}^{H}(u) \mathbf{R}(u) \mathbf{w}(u)$. The covariance matrix is typically estimated by averaging over $\tilde{L}<L$ range bins. If the $l^{t h}$ fast time sample sample is $t_{l}, \hat{\mathbf{R}}(u)$ is known as the sample matrix estimate with $\mu \mathrm{dB}$ of diagonal loading,

$$
\hat{\mathbf{R}}(u)=\frac{1}{\tilde{L}} \sum_{l=1}^{\tilde{L}} \mathbf{z}\left(t_{l}, u\right) \mathbf{z}^{H}\left(t_{l}, u\right)+\mu \mathbf{I}_{N} \in \mathcal{C}^{N \times N}
$$

It is assumed that techniques as described in [1] can be used to get a interference plus noise only estimate of the covariance matrix and hence the spatial vector $\mathbf{z}(\cdot)$ is obtained from signal free data. The diagonal loading acts to improve the robustness by smoothing the adaption via compression of the eigenvalues of the covariance matrix [7]. The constrained optimisation problem is solved using Lagrange multipliers to find the weight vector [8],

$$
\mathbf{w}(u)=\hat{\mathbf{R}}^{-1}(u) \mathbf{C}(u)\left[\mathbf{C}^{H}(u) \hat{\mathbf{R}}^{-1}(u) \mathbf{C}(u)\right]^{-1} \mathbf{d}
$$

\section{PERFORMANCE MEASURES}

The adaptive performance is measured by the amount of interference energy remaining after cancellation. If $y_{\text {ideal }}(t, u)$ is the output signal with no interference present, then the Residual Interference to Noise Ratio (RINR) is given by,

$$
R I N R(t, u)=\frac{|y(t, u)|^{2}}{\left|y_{\text {ideal }}(t, u)\right|^{2}}
$$

For comparisons in this paper, the RINR is measured directly after adaption and is averaged over all the rangebins and pulses.

A second measure of performance is the Signal Distortion Ratio (SDR) post image formation. Let $Y\left(x_{f}, y_{g}\right)$ denote the adapted images for pixels $f=1 \ldots F, g=$ $1 \ldots G$. Correspondingly, let $D\left(x_{f}, y_{g}\right)$ denote the ideal image with no jammer added. The SDR is then defined as

$$
S D R=\frac{\sum_{f, g}\left|D\left(x_{f}, y_{g}\right)\right|^{2}}{\sum_{f, g}\left|Y\left(x_{f}, y_{g}\right)-D\left(x_{f}, y_{g}\right)\right|^{2}}
$$

As the final image is the key result, it is the SDR which is the most appropriate comparison for this work.

\section{SIMULATED RESULTS}

The most common use of the weight vector in Equation 10 is to minimise the beamformer variance, also called the Minimum Variance Distortionless Response (MVDR). This involves constraining the look direction to be unity by substituting $\mathbf{C}(u)=\mathbf{s}(u)$ and $\mathbf{d}=1$ into Equation 10. This technique provides good interference cancellation with sharp nulls at each interference direction. Unfortunately, as the hot-clutter scatterers change from pulse to pulse, so does the interference direction for each corresponding patch. As range profiles are built up with each pulse, coherent modulations are formed over the entire 
processing interval. Then after image formation, the Signal Distortion Rate (SDR) of the MVDR adaption ends up being no better than the conventional beamformer! These secondary effects have been studied previously for target detection in airborne radar [3] and require modifications to the single constraint optimisation.

Improved 'robust' adaption is therefore required and can be implemented by using extra constraints in the weight vector formulation [8]. The goal of these techniques is to reduce potential target signal suppression and hence improve the final SDR. The first of these methods fixes a number of amplitudes around the steering direction. A three point constraint is used in this example, with the amplitude constraints defined as

$$
\mathbf{C}(u)=[\mathbf{s}(u-\delta(u)), \mathbf{s}(u), \mathbf{s}(u+\delta(u))]^{T} \quad ; \quad \mathbf{d}=[1,1,1]^{T}
$$

The optimal choice of $\delta(u)$ will depend on the level of target signal suppression and its effect on the SDR. Also the contribution of the diagonal loading will act to smooth any holes that form from the amplitude constraint. Figure 4 shows the variation of $\delta(u)$ from 0 to 15 degrees with $\mu=25 \mathrm{~dB}$ of diagonal loading and $\tilde{L}=5 \mathrm{~N}$ averages for the sample matrix estimate. Based on the SDR, the best choice of $\delta(u)$ gives $6.9 \mathrm{~dB}$ at 10 degrees. At this point the RINR is also at a minimum of $5 \mathrm{~dB}$.

A second method to reduce potential target signal suppression requires first and/or second order derivatives to be zero in the steering direction. The form of the derivatives is based on differentiating the steering vector in Equation 2.

$$
\begin{aligned}
\frac{\partial s_{n}(u)}{\partial \theta(u)} & =s_{n}(u)\left[-j d_{n} k_{c} \cos \theta(u)\right] \\
\frac{\partial^{2} s_{n}(u)}{\partial \theta^{2}(u)} & =j d_{n} k_{c}\left[s_{n}(u) \sin \theta(u)-\frac{\partial s_{n}(u)}{\partial \theta(u)} \cos \theta(u)\right]
\end{aligned}
$$

The derivative constraints can then be written as,

$$
\mathbf{C}(u)=\left[\mathbf{s}(u), \frac{\partial \mathbf{s}(u)}{\partial \theta(u)}, \frac{\partial^{2} \mathbf{s}(u)}{\partial \theta^{2}(u)}\right]^{T} \quad ; \quad \mathbf{d}=[1,0,0]^{T}
$$

The form of the first order only constraint doesn't include the third term in either $\mathbf{C}(u)$ or d. Results in Figure 4 show that the first order constraint yields the best SDR at $6.5 \mathrm{~dB}$ and an RINR of 5.7dB. The second order constraint performs worse than the MVDR constraint as it reduces the interference as well as the target signal suppression. Figure 3 shows a comparison between the synthetic SAR image with no jamming, jamming and the recovered image using first-order derivative constraints. The image size is $(F, G)=169 \times 150$ pixels.

\section{CONCLUSIONS}

The use of amplitude and/or derivative constraints with spatial only adaption has improved the SDR of the MVDR adaption by up to $2.6 \mathrm{~dB}$. This is also reflected in the RINR which indicates that more of the interference has been cancelled. Further work using fast-time taps should provide an improvement for this situation.

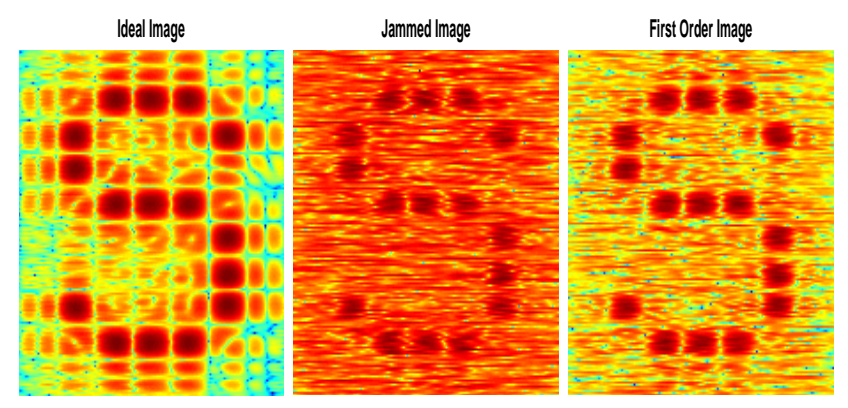

Fig. 3. Ideal SAR Image, Jammed Image, and Adapted Image using First order Derivative Constraints
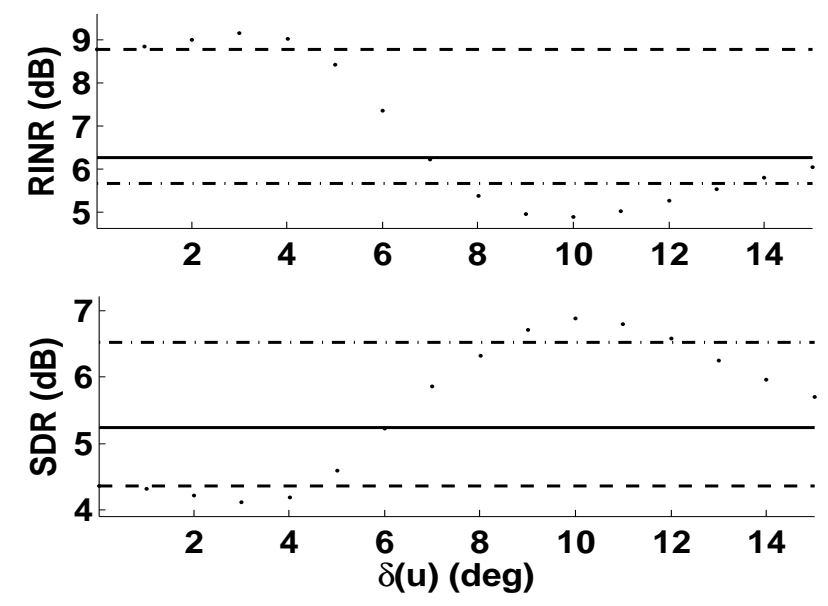

Fig. 4. Varying $\delta(u)$ : (-) MVDR, $(\cdots)$ Amplitude, (-.-) First Order Deriv., (- -) Second Order Deriv.

\section{REFERENCES}

[1] J. Ward, "Space-Time Adaptive Processing for Airborne Radar," Technical report 1015, Lincoln Labs. MIT, 1994.

[2] R. A. Gabel, S. M. Kogon, and D. J. Rabideau, "Algorithms for Mitigating Terrain-Scattered Interference," in Electronics \& Communication Engineering Journal. 1999, Lincoln Labs. MIT.

[3] D. Rabideau, "Clutter and jammer multipath cancellation in airborne adaptive radar," IEEE Proceedings on Aerospace and Electronic Systems, vol. v36, no. 2, April 2000.

[4] P Beckman, The Scattering of Electromagnetic Waves from Rough Surfaces, Pergamon Press Ltd., 1963.

[5] R. L. Fante, "Cancellation of Specular and Diffuse Jammer Multipath using a Hybrid Adaptive Array," in IEEE Transactions on Aerospace and Electronic Systems. 1991, Mitre Corp. Bedford MA USA.

[6] L. Rosenberg and D. Gray, "Multichannel SAR Imaging using Wavefront Reconstruction," in International Radar Symposium Proceedings, 2004.

[7] J. Ward, H. Cox, and S.M. Kogon, "A Comparison of Robust Adaptive Beamforming Algorithms," in Asilomar Conference on Signals, Systems and Computers, 2003, vol. 2.

[8] D.H. Johnson and D.E. Dudgeon, Array Signal Processing - Concepts and Techniques, Prentice Hall Inc., 1993. 